IDDF2019-ABS-0091 COMPARISON OF CLINICAL CHARACTERISTICS AND QUALITY OF LIFE ON ROME IV VS ROME III CRITERIA FOR IRRITABLE BOWEL SYNDROME IN CHINA: A MULTI-CENTER STUDY

${ }^{1}$ Xin Yao*, ${ }^{2}$ Yunsheng Yang, ${ }^{1}$ Shutian Zhang, ${ }^{3}$ Lihong Cui, ${ }^{4}$ Nan Wang, ${ }^{5}$ Qian Zhang ${ }^{1}$ Department of Gastroenterology, Beijing Friendship Hospital, Capital Medical University, National Clinical Research Center for Digestive Disease, Beijing Digestive Disease Center, Beijing Key Laboratory for Precancerous Lesion of Digestive Disease, China; ${ }^{2}$ Institute of Digestive Diseases, Chinese PLA General Hospital, Chinese PLA Medical Academy, China; ${ }^{3}$ Department of Gastroenterology, Naval General Hospital, China; ${ }^{4}$ Department of Gastroenterology, Bethune International Peace Hospital, China; ${ }^{5}$ Clinical Epidemiology and EBM Unit, National Clinical Research Center for Digestive Disease, Beijing Friendship Hospital, Capital Medical University, China

\subsection{6/gutjnl-2019-IDDFabstracts. 140}

Background The Rome criteria for irritable bowel syndrome (IBS) have been revised to Rome IV criteria in 2016. The aim of this multicenter study was to compare the clinical characteristics and quality of life for IBS on Rome IV vs. Rome III criteria in China.

Methods Nine hundred and forty IBS outpatients from three tertiary hospitals in China were included in this study. Diagnostic criteria were based on Rome III or Rome IV criteria. The demographic characteristics, IBS symptoms, coexisting upper gastrointestinal symptoms and extraintestinal symptoms of the IBS patients were recorded. Of the total IBS patients, 518 completed the evaluation of psychological status and health-related quality of life (HRQOL). Psychological problem was evaluated using the validated Hospital Anxiety and Depression scale (HAD), self-rating anxiety scale (SAS) and self-rating depression scale (SDS). HRQOL was assessed by 36-item Short-Form Health Survey (SF-36).
Results Among the 940 IBS patients, 935 patients met the Rome III criteria, 498 patients met the Rome IV criteria, and 493 met both sets of criteria. Among the 935 patients meeting the Rome III criteria, 493 met both sets of criteria (group 1) and 442 met Rome III criteria only (group 2). Subjects in group 1 were more often male and reported higher abdominal pain scores, defecation urgency and symptom frequency compared with patients in group $2(P<0.05)$. Furthermore, the patients in group 1 experienced more regurgitation, nausea, epigastrium pain or burning $(P<0.05)$ and presented with greater rates of fatigue, insomnia, lumbodorsal pain, dizziness, chest distress, headache, weight loss $(\geq 4.5 \mathrm{Kg})$ and chest pain compared with those in group 2(figure 1 ), $P<0.05$ ). The patients in group 1 scored lower in most of the domains of SF-36, mental component summary, physical component summary and total SF-36 $(P<0.05)$ than those in group 2 while the anxiety and depression scores were not statistically different $(P>0.05)$. (figure 1)

Conclusions IBS population meeting Rome IV \& III criteria presented higher abdominal pain, defecation urgency, symptom frequency, upper gastrointestinal and extraintestinal symptoms and poorer HRQOL than IBS patients meeting Rome III only.

\section{IDDF2019-ABS-0096 THE USE OF ELOBIXIBAT AND LACTULOSE FOR BOWEL PREPARATION FOR COLON CAPSULE ENDOSCOPY}

Naoki Hotta*. Masuko Memorial Hospital Department of Gastroenterology, Japan

10.1136/gutjnl-2019-IDDFabstracts. 141

Background Colon Capsule Endoscopy was approved for reimbursement under the national health insurance system of Japan






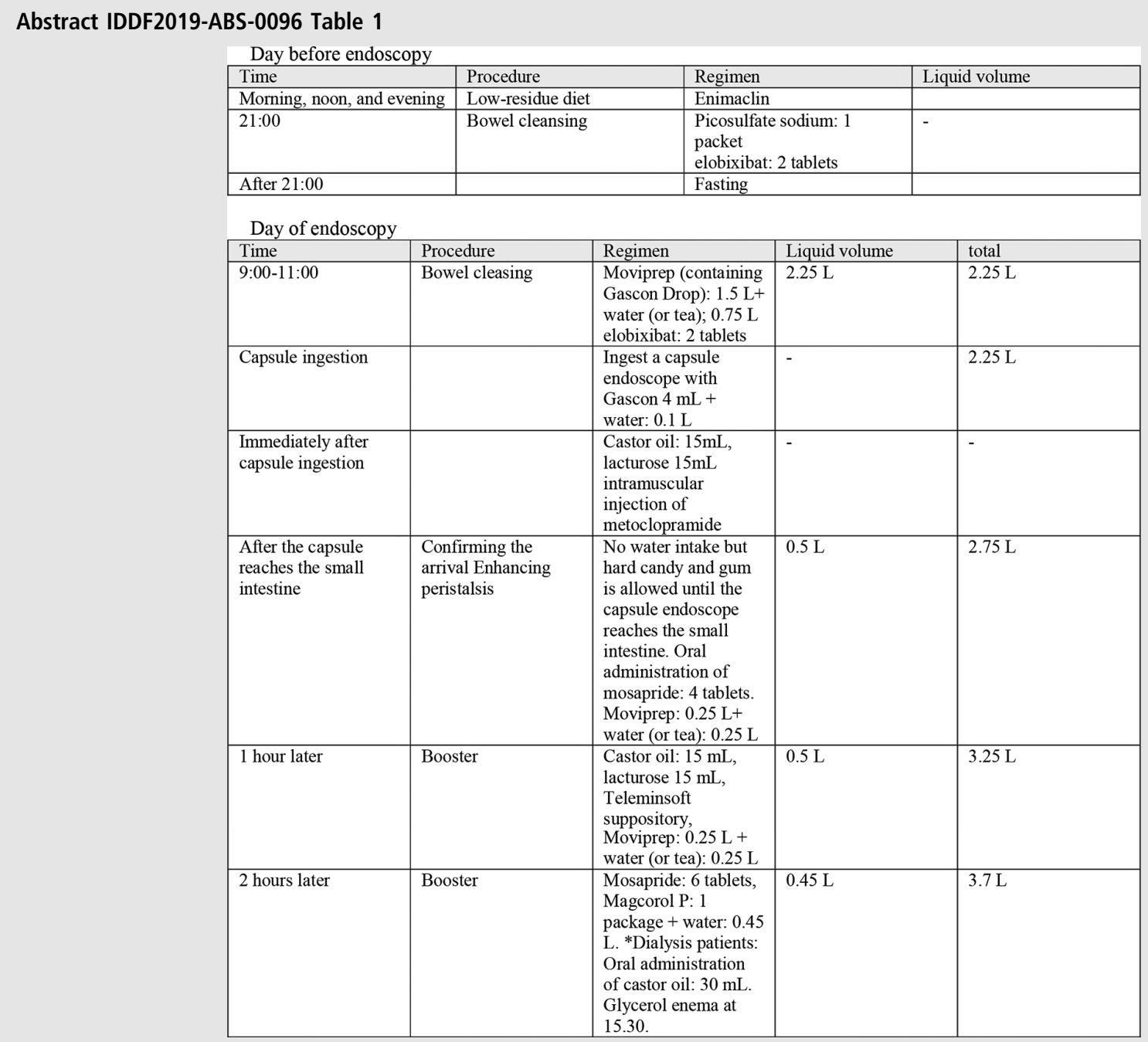

in 2014. For this reason, a detailed examination by capsule endoscopy appears to be useful. However, the capsule excretion rate after recommended bowel preparation reportedly ranges from $70 \%$ to $90 \%$.In this study, we evaluated the efficacy of bowel preparation with Elobixibat and lactulose for improving the capsule excretion rate.

Methods This study included 46 patients who underwent colon capsule endoscopy at Masuko Memorial Hospital since March 2018. The recommended protocol of bowel preparation was modified(with Elobixibat and lactulose) and success rates of completing entire colon observation were compared. Elobixibat(Goofice: EA pharma, Japan) at the beginning of the day before enforcement and the enforcement. Lactulose(Morinaga, Japan) was administered immediately after capsule ingestion and as a booster at one and two hours later. This study was approved by the ethics committee of my hospital, while we obtained written consent from the participants after providing a thorough explanation of the contents and methods of this study. (table 1)

Results The success rate of completing entire colon observation was 43patients were 46(93.4\%)in this study(23 dialysis patients and 23 non-dialysis patients). The success rates were 23 patients included $22(95.6 \%)$ in the non-dialysis patients and $(21 / 23)$ in the dialysis patients.
Conclusions Although the number of patients receiving preparation with Elobixibat and lactulose was small, entire colon observation was successful even in the dialysis patients. Thus, Elobixibat and lactulose appear to be useful for bowel preparation for colon capsule endoscopy.

\section{IDDF2019-ABS-0098 THE EFFECT OF PREDICTABLE NURSING MEASURES ON COMMON BILE DUCT STONE PATIENTS IN POST ERCP COMPLICATIONS}

Fang Wang*, Wen-Hui Tan, Ling He, Fang Lv. Guangdong Second Provincial General Hospital, China

\subsection{6/gutjnl-2019-IDDFabstracts. 142}

Background To explore the effect of predictable nursing measures on preventing post ERCP complications of common bile duct stone patients.

Methods 100 common bile duct stone patients were enrolled in our study. Patients were randomly divided into two groups. Routine nursing was performed in control group $(n=50)$, while predictable nursing was performed in observation group $(n=50)$. Data on overall survival, technical failure, nursing 